Apidologie, 1972, 3 (3) 263-273.

\title{
RÉPARTITION DES VOLS D'ABEILLES SAUVAGES DANS QUELQUES VERGERS DE LA RÉGION PARISIENNE AU COURS DES ANNÉES 1969 ET 1970
}

\author{
Verteilung der Flüge von Wildbienen in einigen Obstanlagen \\ des Pariser Raumes in den Jahren 1969 und 1970
}

\author{
Jacques CHANSIGAUD \\ Laboratoire d'Éthologie et d'Écologie des Insectes, I.N.R.A., \\ 91 - Bures-sur-Yvette
}

\begin{abstract}
SUMMARY
DISTRIBUTION OF THE FLIGHTS OF WILD BEES IN SOME ORCHARDS NEAR PARIS FROM 1969 TO 1970
\end{abstract}

As a contribution to the general study of pollination, carried out in the Laboratory of insect Ethology and Ecology, the author has studied the distribution of different species of wild bees in some orchards in the surroundings of Paris. The bees observed belonged mainly to the genus Andrena. The number of species varies in relation to the places of investigation. For instance, in La Norville near Arpajon, south of Paris, we determined 9 species foraging on the blossoms of Golden delicious apple, out of a total number of 16 species in all the orchards. The most frequently observed Andrena are $A$. hoemorrhoa $F a b r, A$. armata Gmel, $A$. carantonica Perez. We attach great importance to the dates of capture of the different species. In fact, we believe that synchronism between pollinating activity and periods of blossoming is dependent on duration of flights. Accordingly captures were effected successively on pear, dessert apple, cider apple, and it appeared that three species, $A$. hoemorrhoa, $A$. carantonica, $A$. armata have the longest flights. The study of the first two is interesting, because they were collected from the blossoming of pears.

The percentage of wild bees reaches 5 to $10 \%$ of the total number of bees registered in the orchards, honeybees included. 


\section{RESUMÉ}

Dans le cadre général des recherches sur la pollinisation effectuées au Laboratoire d'Éthologie et d'Écologie des Insectes, l'auteur a étudié la répartition des vols de différentes espèces d'abeilles_sauvages dans quelques vergers de la région parisienne.

Les abeilles rencontrées appartiennent surtout au genre Andrena. Le nombre des espèces varie en fonction du site. Ainsi, à La Norville, près d'Arpajon, au sud de Paris, on a déterminé 9 espèces butinant sur les fleurs des pommiers “ Golden 》 sur un total de 16 espèces pour l'ensemble des vergers. Les Andrènes le plus souvent observées sont $A$. hoemorrhoa Fabr., A. armata Gmel., A. carantonica Perez.

Nous attachons une importance particulière aux dates de capture des différentes espèces. En effet, nous pensons que le synchronisme entre l'activité pollinisatrice et les dates de floraison est fonction de la durée plus ou moins longue des périodes de vol. D'après les captures effectuées successivement sur poiriers, sur pommiers à couteau et pommiers à cidre, $A$. hoemorrhoa, $A$. armata, $A$. carantonica, sont les abeilles sauvages qui ont les périodes de vol les plus longues. $A$. hoemorrhoa, $A$. carantonica, sont intéressantes à étudier car elles ont été capturées précocement sur les fleurs des poiriers.

Le pourcentage des abeilles solitaires est compris entre 5 et $10 \%$ du total des Apides dénombrés, abeilles domestiques inclues.

\section{INTRODUCTION}

Apis mellifica $L$ et les Apides sauvages appartenant aux genres Bombus, Andrena, Malictus et Osmia sont les principaux pollinisateurs des arbres fruitiers. Cependant, les Bourdons et les abeilles domestiques ont une activité de butinage supérieure à celle des abeilles sauvages, ne serait-ce que par le nombre de fleurs visitées par unité de temps. Les auteurs reconnaissent néanmoins aux abeilles solitaires des qualités que n'ont pas les abeilles domestiques au cours du butinage. En effet, contrairement à ces dernières, elles n'humidifient pas le pollen récolté et le répartissent sur une plus grande surface de leur corps, notamment sur les brosses des fémurs et celles des trochanters. Le pollen se détache donc plus facilement et en plus grande quantité lors du passage des insectes sur les fleurs. Le désavantage découlant de leur lenteur de travail pourrait être alors compensé par une augmentation de l'efficacité pollinisatrice. D'autre part, il est important de noter que certaines abeilles sauvages ont un seuil thermique d'activité inférieur à celui d' $A$. mellifica.

Les aspects positifs que nous venons d'évoquer rapidement au sujet du comportement de butinage des abeilles solitaires laissent supposer que certaines d'entre elles peuvent présenter un intérêt agronomique. C'est pourquoi nous mettons en place une série d'expérimentations à caractère éco-éthologique dont les premières ont pour objet d'établir de façon précise le rôle complémentaire joué par ces Apides sur le plan de la pollinisation des arbres fruitiers, notamment de ceux dont la floraison est précoce. 


\section{MATERIEL ET METHODES}

En 1969, les observations ont commencé le 16 avril, au début de la floraison des poiriers; elles se sont poursuivies sur les pommiers à couteau du 28 avril au 9 mai.

En 1970, commencés sur les poiriers le 27 avril, les relevés se sont échelonnés jusqu'au 4 juin, date de la fin de la floraison des pommiers à cidre.

Les variétés fruitières intercompatibles choisies pour les comptages étaient les suivantes :

- pour les poiriers : William et Doyenne du Comice,

- pour les pommiers à couteau : Golden delicious et Jonathan,

- pour les pommiers à cidre : Doux Veret de Carrouge, Douce Moen et Egyptia.

En 1969, les observations ont été entreprises dans un seul verger situé au sud de Paris, à La Norville, près d'Arpajon.

En 1970, cinq plantations localisées à l'ouest de Paris dans la région de Versailles ont servi aux observations.

En 1969, l'après-midi de préférence, nous avons capturé le plus grand nombre possible d'abeilles solitaires butinant sur les fleurs des pommiers a Golden » en nous déplaçant pendant une heure le long des haies fruitières, sans repasser deux fois au même endroit. Les insectes étaient capturés directement sur les fleurs et tués pour être déterminés.

L'année suivante, les observations ont été faites pendant une heure entre 10 et $16 \mathrm{~h}$ en alternant chaque jour les horaires des comptages dans chacune des plantations de façon à éviter qu'un lieu donné soit visité constamment à la même heure. Cette méthode a l'avantage de donner un meilleur éventail des espèces présentes, étant donné la prolongation des observations pendant une heure. Par contre, il est certain que sur un laps de temps aussi long, les chances de recenser plus d'une fois un insecte ne sont pas négligeables. C'est d'ailleurs la raison pour laquelle on effectue les comptages sur une période beaucoup plus courte. De ce fait, les effectifs ne peuvent être considérés comme absolus. Nous utilisons donc le mot « fréquence » pour désigner la quantité d'abeilles observées, car, dans le cas présent, ce terme intégre un facteur “ mobilité des insectes » qui favorise l'abeille domestique plus mobile que les abeilles solitaires. Ainsi, d'après les dénombrements effectués pendant une heure, nous pouvons connaître en chaque endroit la fréquence relative des abeilles d'une espèce déterminée ou d'un groupe d'espèces par rapport au total des fréquences observées (toutes espèces comprises) pendant le même temps et sur le même parcours.

La méthode utilisée en 1970 est visuelle; il n'y a pas de capture systématique des insectes recensés mais seulement quelques prélèvements faits au hasard qui permettent d'obtenir des renseignements, espèce par espèce sur la période théorique de vol des abeilles. Cette période, pour une espèce donnée, débute avec la première observation qui a pu être faite et s'achève avec la dernière, soit toujours sur la même variété fruitière, soit sur l'ensemble des variétés étudiées. La connaissance de la période théorique de vol d'une abeille fournit une première indication quant à l'intérêt agronomique de cette espèce et constitue une base de sélection des insectes susceptibles de faire l'objet d'une expérimentation ultérieure.

On sait que les Halictes et les Andrènes nidifient en terre dans des sites qu'elles choisissent en fonction de la nature du sol. Il n'est donc pas inutile de procéder à quelques notations à ce sujet. Dans la région étudiée, encore relativement boisée, les arbres fruitiers sont plantés de préférence sur des terrains sableux ou limoneux (limon argileux). L'alternance de ces deux types de sol est d'ailleurs fréquente dans un même lieu. Ainsi, à La Jonction (St-Germain-en-Laye), à l'orée de la forêt de Marly, le sol du verger est un limon argileux tandis qu'à proximité on trouve des marnes et du sable. A La Norville, toute la périphérie de la plantation est sableuse alors que la partie centrale est par endroits argileuse. 


\section{RESULTATS}

Les abeilles sauvages capturées au cours des deux années 1969 et 1970 appartiennent en majorité au genre Andrena. Toutefois les dénombrements visuels de 1970 font apparaître aussi un petit nombre d'abeilles des genres Halictus et Osmia.

Le tableau 1 regroupe les données obtenues sur poiriers et le tableau 2 sur pommiers.

Pour l'ensemble des vergers et pour les deux années d'observations nous avons capturé 16 espèces d'Andrènes. Treize ont été prises sur les arbres à fruits de table et les trois dernières de la liste suivante, uniquement sur les fleurs des pommiers à cidre :

Andrena armata Gmel., A. bimaculata Kirby, A. carantonica Perez, $A$. cineraria L., $A$. chrysoscelles Kirby, A. dorsata Kirby, A. flavipes Panz., $A$. gravida Imh., $A$. helvola L., $A$. hoemorrhoa Fabr., $A$. mitis Schm., $A$. minutula Kirby, A. nitida Müll., A. florea F., $A$. ovatula Kirby, $A$. strohmella Stock.

Si l'on considère qu'il existe en France 250 espèces du genre Andrena (Platea d-Quenu, 1972), la quantité ci-dessus représente 6,4\% des espèces existantes. Les espèces rencontrées diffèrent d'un site à l'autre. Sur les arbres à fruits de table, c'est à La Norville en 1969 et à La Jonction en 1970 que nous avons trouvé le plus d'Andrènes. A La Norville, $A$. hoemorrhoa et $A$. armata sont les abeilles sauvages les plus nombreuses. Sur un total de 110 insectes récoltés au cours de 11 observations de une heure, leurs nombres respectifs sont de 44 et de 22 (Fig. 1).

En 1970, pour chacune des plantations fruitières nous indiquons uniquement (Tableau 3 ) les espèces capturées et le total de celles-ci. Il est intéressant de constater que beaucoup d'entre elles sont encore en activité sur les fleurs des pommiers à cidre.

Les relevés faunistiques effectués montrent qu' $A$. armata, $A$. carantonica et $A$. hoemorrhoa sont les abeilles qui ont été le plus souvent observées.

Les choix que nous nous proposons de faire en vue d'une expérimentation ultérieure reposent, comme nous l'avons signalé plus haut sur la longueur et la position dans le temps de la période théorique de vol. Cette période est estimée d'après les captures effectuées successivement sur les poiriers, les pommiers à couteau et les pommiers à cidre (Fig. 2). En effet, il est évident que le synchronisme entre l'activité pollinisatrice et les dates de floraison est fonction de la durée plus ou moins longue des périodes de vol. C'est pourquoi, 


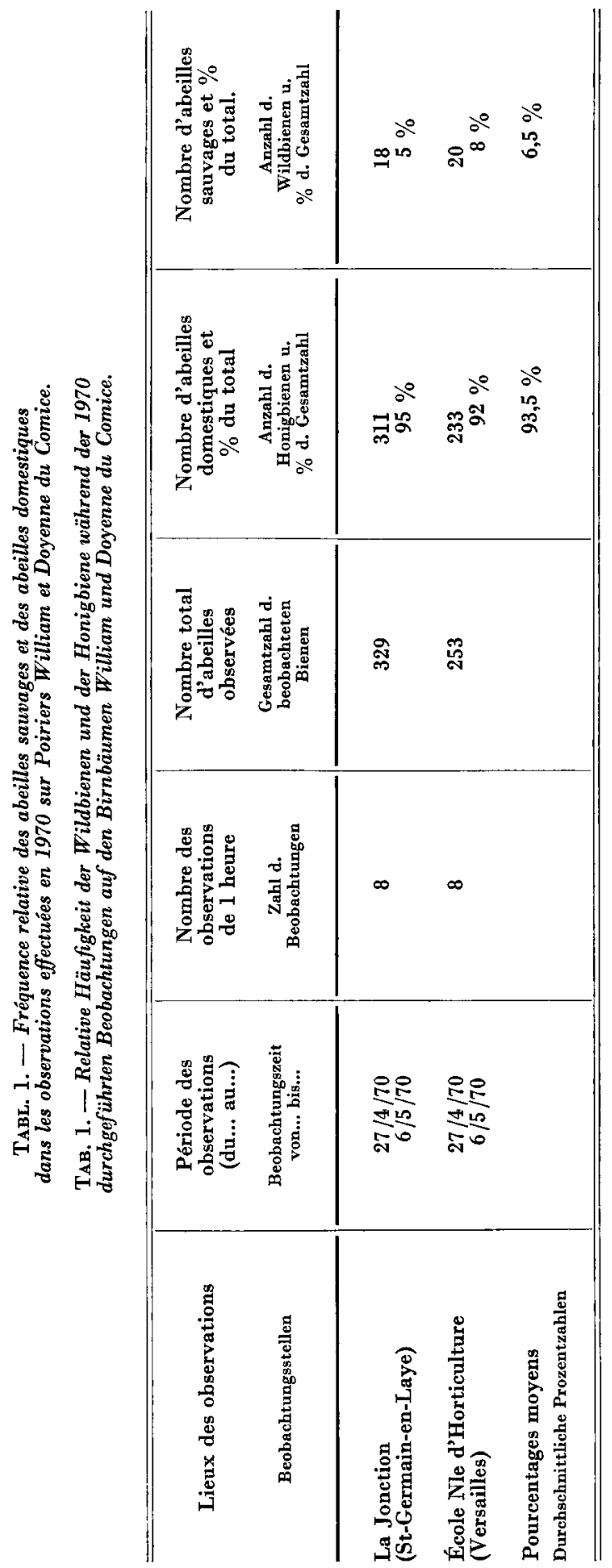


J. Chansigaud

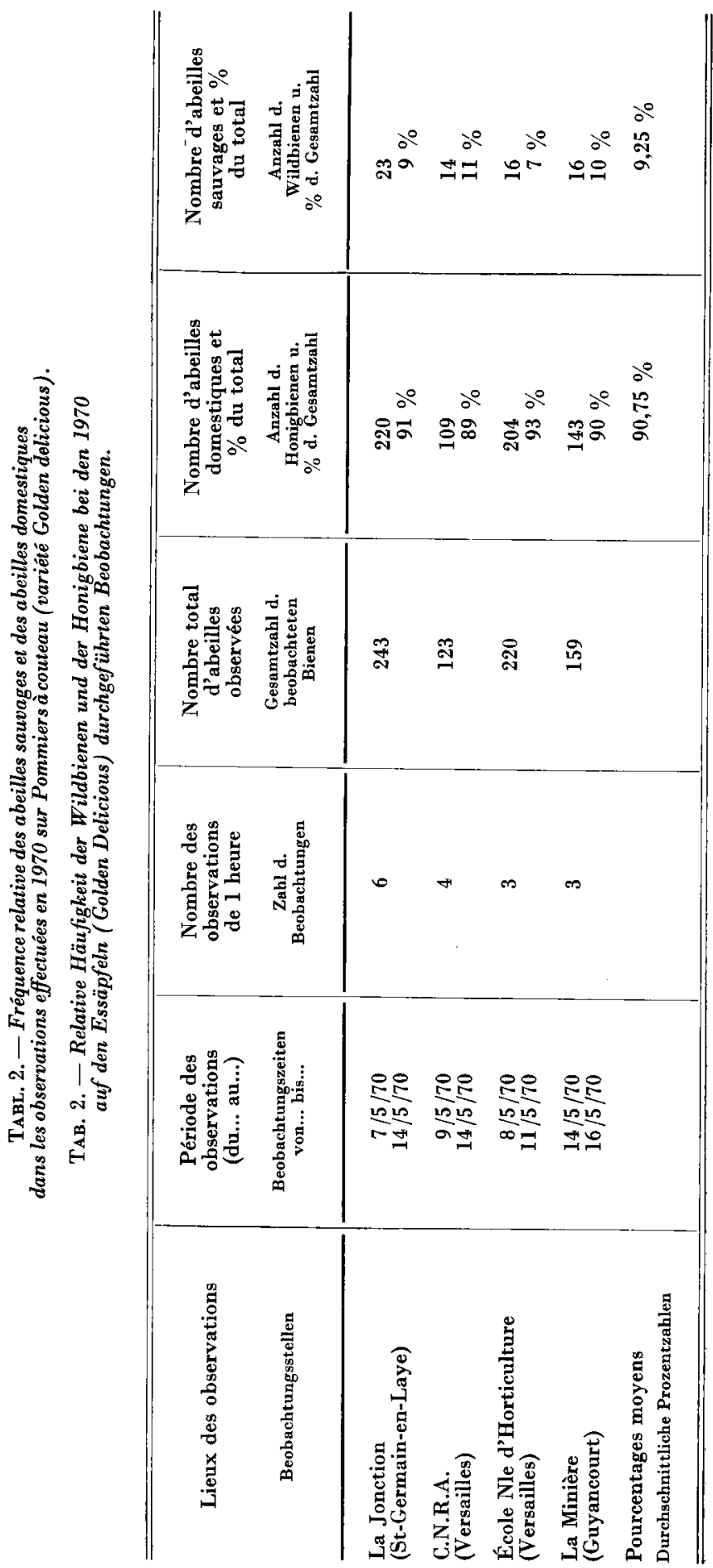


dans l'immédiat, nous pensons intéressant d'orienter nos recherches vers les trois espèces $d$ 'Andrènes citées au paragraphe précédent, dont la période d'activité recoupe les dates de floraison des poiriers, des pommiers à couteau ainsi que des pommiers à cidre les plus précoces.

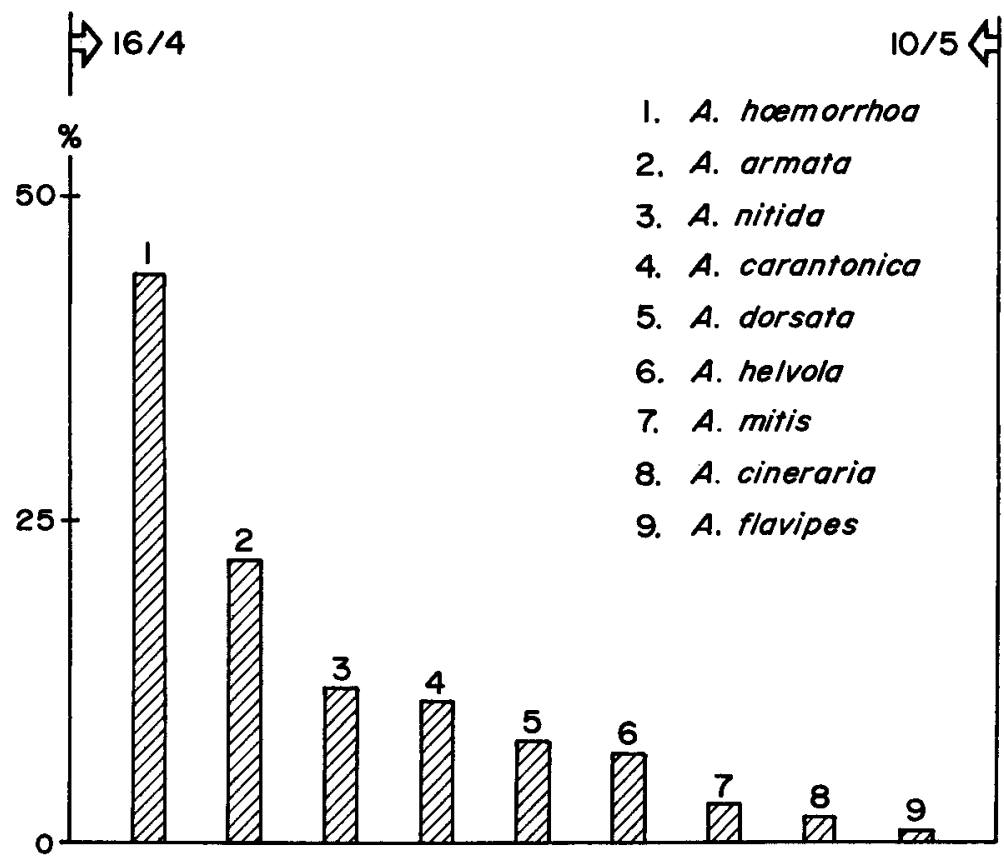

Fig. 1. - Pourcentage des différentes espèces d'Andrènes rêcoltées à La Norville en 1969. $16 / 4,10 / 5=$ Période de floraison des poiriers et des pommiers

Aв8. 1. - Prozentsatz der verschiedenen Andrena-Arten, die in La Norville im Jahre 1969 angetroffen wurden. $16 / 4,10 / 5=$ Blütezeiten der Birnen und Äpfel.

\section{CONCLUSIONS}

Dans la partie du bassin parisien que nous avons prospectée pendant deux années consécutives on peut, avec une bonne approximation estimer le pourcentage des abeilles sauvages butinant sur les fleurs des arbres fruitiers à $5-10 \%$ et celui des abeilles domestiques à $90-95 \%$. Ce dernier pourcentage est conforme aux observations faites notamment par Davis (1925), Mac Daniels et Heinicke (1929), Filmer (1941), Wafa et Ibrahim (1957), Dyce (1958).

La diversité des espèces que nous avons trouvées est plus ou moins marquée selon les vergers. C'est à La Norville que nous avons obtenu le plus large éven- 
tail d'abeilles. Cette diversité est due vraisemblablement aux conditions de reproduction qui sont offertes à ces insectes : ressources alimentaires, sol favorable à la nidification. A ce propos on remarque (Tableau 3) qu' $A$. carantonica est surtout présente dans les sites où le sol est constitué de limon argileux : La Minière, La Jonction, La Norville. Cette observation rejoint celle de KOCOUREK (1966).

TABL. 3. - Espèces du genre Andrena rencontrées dans les plantations fruitières en 1969 et 1970. Observations effectuées sur Poiriers, Pommiers à couteau et Pommiers à cidre.

TAB. 3. - Andrena-Arten, die 1969 und 1970 in den Obstanlagen angetroffen wurden. Beobachtungen auf Birnen und Tafel- $u$. Kelteräpfeln

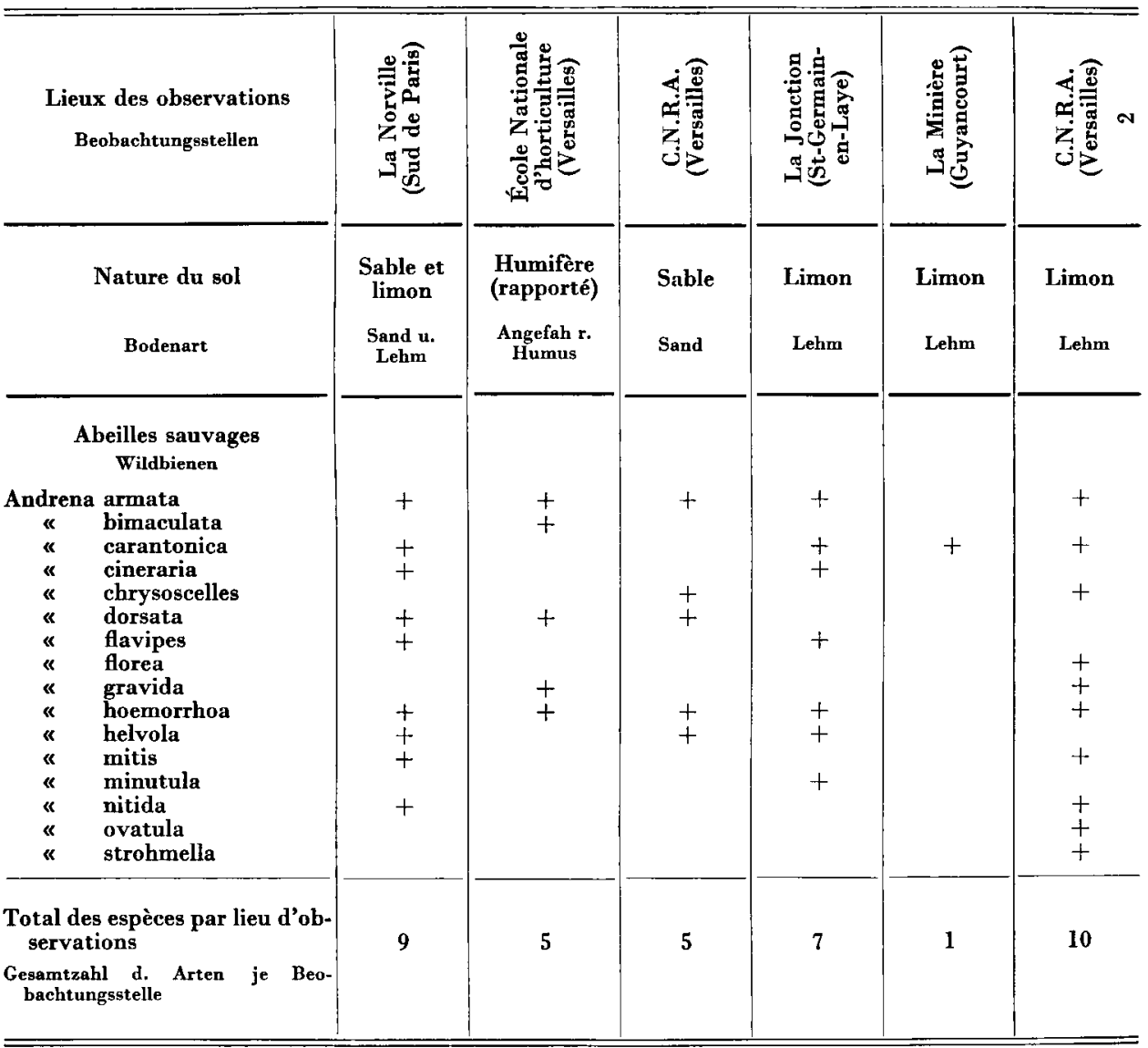

Les Andrènes sélectionnées pour leur intérêt agronomique, $\boldsymbol{A}$. carantonica, $A$. hoemorrhoa et $A$. armata, sont fréquemment citées dans la littérature comme pollinisatrices des arbres fruitiers, en particulier par ChAmbers (1946). L'auteur 


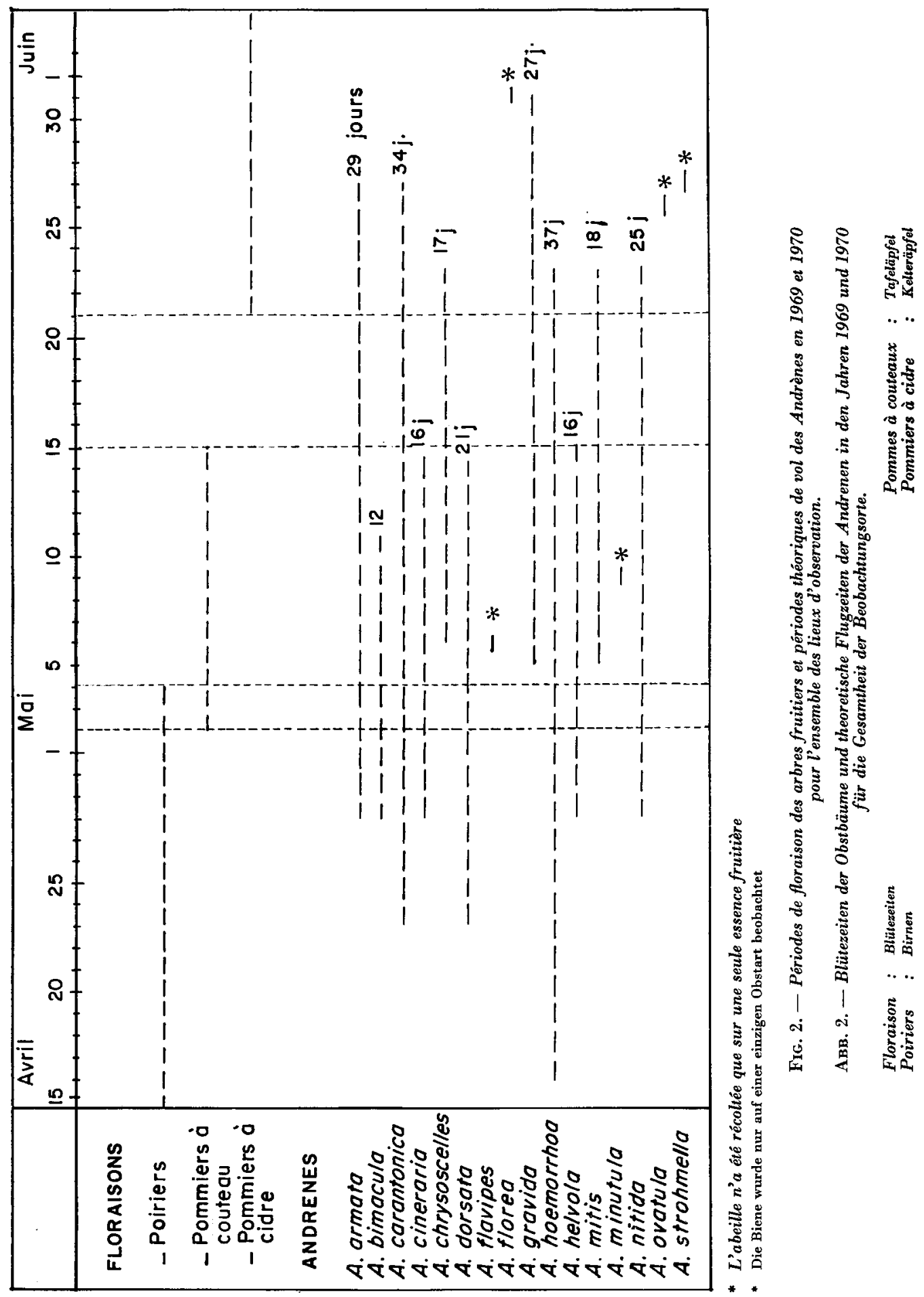


a fait à ce sujet d'intéressantes observations sur les charges de pollen d'A. hoemorrhoa. Il reste bien entendu que le choix effectué pour nos recherches ne préjuge en rien de la valeur pollinisatrice des autres espèces citées dans le présent travail.

Reçu pour publication en avril 1972.

Eingegangen in April 1972.

\section{REMERCIEMENTS}

Nous remercions M. Desmier de Chenon de la Station de Zoologie du C.N.R.A. qui a bien voulu se charger de la détermination des Apides.

\section{ZUSAMMENFASSUNG}

Apis mellifica sowie Wildbienen der Gattungen Bombus, Andrena, Halietus und Osmia sind es hauptsächlich, die die Obstblüten bestäuben. Obwohl Apis mellifica ihres beträchtlichen Bestandes wegen stets die Hauptbestäuberin darstellt, ist es doch von Interesse, auch die Rolle, die möglicherweise Wildbienen spielen, zu untersuchen. Ihre zahlenmässige Unterlegenheit wird bis zu einem gewissen Grade durch Besonderheiten ihres Sammelverhaltens ausgeglichen, wodurch diese Bienen unter Umständen zu guten Bestäubern werden können.

Verf. hat Wildbienen-Populationen in einigen Obstanlagen des Pariser Raumes beobachtet, um festzustellen, welche Arten ein gründliches Studium im Rahmen der vom Laboratorium für Ethologie und Ökologie der Insekten durchgeführten Untersuchungen über die Bestäubung verdienen.

Die Untersuchungen wurden 1969 und 1970 während der Birnen- und Apfelblüte (Tafela. Kelterobst) durchgeführt. Die Berechnung der zu vergleichenden Insekten wurde folgendermassen vorgenommen : Von Apis mellifica und den anderen Bienen (hauptsächlich Andrena) wurden täglich während einer Stunde in den verschiedenen Obstanlagen alle Individuen gezählt, die auf den Blüten der Obstbäume sammelten. Dabei bewegte sich der Beobachter gleichmässig an den Baumreihen entlang, ohne die gleiche Stelle zweimal zu betreten. Das Einfangen zur genauen Bestimmung der Wildbienen erfolgte willkürlich. Die Bodenart der Obstgärten und der Umgebung wurde untersucht, um die Nistbedingungen der im Boden nistenden Arten kennzulernen.

Die Ergebnisse beziehen sich einerseits auf die Häufigkeit der verschiedenen Arten, die angetroffen wurden (Tab. 1 u. 2) und auf die Zahl der in den verschiedenen Obstgärten gezählten Arten (Tab. 3), andererseits auf den Prozentsatz der verschiedenen Andrena-Arten im Süden von Paris im Jahre 1969 (Abb. 1) und auf die theoretische Flugperiode der Andrena-Arten, die hauptsächlich angetroffen wurden (Abb. 2).

16 Andrena-Arten $=6,4 \%$ aller in Frankreich bekannten Arten wurden angetroffen.

Andrena armata, $A$. carantonica und $A$. hoemorrhoa wurden am häufigsten beobachtet. Besonders interessant ist die Flugzeit dieser drei Arten; sie überschneidet in günstiger Weise Birnen- und Apfelblüte. Daher scheinen sie für spätere Untersuchungen besonders gut geeignet. 


\section{RÉFERENCES BIBLIOGRAPHIQUES}

Chambers V.-H., 1946. An examination of the pollen loads of Andrena the species that visit fruit trees. J. anim. Ecol., 15, 9-21.

DAvis J.-J., 1925. Beekeeping in relation to agriculture. Bull. Ind. Agric. Exp. Sta. No 129.

Dyce E.-J., 1958. Honeybees and the pollination problem in New-York State. Glean. Bee Cult., 86 (3), 140-143.

FiLmer 1941. Honeybee population and floral competition in New-Jersey orchards. $J$. Econ. Entom., 34, 198-199.

Koxounex M., 1966. Prodromus der Hymenopteren der Tschekoslowakei Acta faun. entom. Musei. Nat. Pragae. 12 Sup. 2 Edit 30. VII 1966.

Mac Daniels L.-H., Heinicke A.-J., 1929. Pollination and other factors affecting the set of fruit with special reference to the apple. Bull. Cornell. Agric. Exp. Sta. No 497.

Puateau-Quenu C., 1972. La biologie des abeilles primitives. Éd. Masson et Cie. Paris. 200 p.

WAFA et Ibrahim, 1957. The honeybee as an important insect for pollination. Bull. Cairo Univ. Fac. Agric. $\mathrm{N}^{\circ} 162$. 